\title{
Predictive Value of Dynamic Peri-Transplantation MRD Assessed By MFC Either Alone or in Combination with Other Variables for Outcomes of Patients with T-Cell Acute Lymphoblastic Leukemia*
}

\author{
Zhi-dong $\mathrm{WANG}^{1 \dagger}$, Yue-wen $\mathrm{WANG}^{1 \dagger}$, Lan-ping XU ${ }^{1}$, Xiao-hui ZHANG ${ }^{1}$, Yu WANG ${ }^{1}$, Huan $\mathrm{CHEN}^{1}$, Yu-hong CHEN ${ }^{1}$, \\ Feng-rong WANG ${ }^{1}$, Wei HAN ${ }^{1}$, Yu-qian SUN ${ }^{1}$, Chen-hua YAN ${ }^{1}$, Fei-fei TANG ${ }^{1}$, Xiao-dong MO ${ }^{1,2}$, Ya-zhe WANG ${ }^{1}$, \\ Yan-rong LIU1 ${ }^{1}$, Kai-yan LIU1', Xiao-jun HUANG ${ }^{1,2,3}$, Ying-jun CHANG ${ }^{1 \#}$ \\ ${ }^{I}$ Peking University People's Hospital and Peking University Institute of Hematology, National Clinical Research Center for \\ Hematologic Disease, Beijing Key Laboratory of Hematopoietic Stem Cell Transplantation, Beijing 100044, China \\ ${ }^{2}$ Research Unit of Key Technique for Diagnosis and Treatments of Hematologic Malignancies, Chinese Academy of Medical \\ Sciences, Beijing 100005, China \\ ${ }^{3}$ Peking-Tsinghua Center for Life Sciences, Beijing 100871, China
}

(C) The Author(s) 2021

\begin{abstract}
Summary: We performed a retrospective analysis to investigate dynamic peri-hematopoietic stem cell transplantation (HSCT) minimal/measurable residual disease (MRD) on outcomes in patients with T-cell acute lymphoblastic leukemia (T-ALL). A total of 271 patients were enrolled and classified into three groups: unchanged negative MRD pre- and post-HSCT group (group A), post-MRD non-increase group (group B), and post-MRD increase group (group C). The patients in group $\mathrm{B}$ and group $\mathrm{C}$ experienced a higher cumulative incidence of relapse (CIR) $(42 \% \mathrm{vs} .71 \%$ vs. $16 \%, P<0.001)$ and lower leukemia-free survival (LFS) $(46 \% v s .21 \%$ vs. $70 \%, P<0.001)$ and overall survival (OS) $(50 \%$ vs. $28 \%$ vs. $72 \%, P<0.001)$ than in group A, but there was no significant difference in non-relapse mortality (NRM) among three groups (14\% vs. $12 \%$ vs. $8 \%, P=0.752)$. Multivariate analysis showed that dynamic peri-HSCT MRD was associated with CIR (HR=2.392, 95\% CI, 1.816-3.151, $P<0.001)$, LFS (HR=1.964, 95\% CI, 1.546-2.496, $P<0.001)$ and OS $(\mathrm{HR}=1.731,95 \% \mathrm{CI}, 1.348-2.222, P<0.001)$. We also established a risk scoring system based on dynamic peri-HSCT MRD combined with remission status pre-HSCT and onset of chronic graftversus-host disease (GVHD). This risk scoring system could better distinguish CIR ( $\mathrm{c}=0.730)$ than that for pre-HSCT MRD ( $\mathrm{c}=0.562)$, post-HSCT MRD $(\mathrm{c}=0.616)$ and pre- and post-MRD dynamics $(c=0.648)$. Our results confirm the outcome predictive value of dynamic peri-HSCT MRD either alone or in combination with other variables for patients with T-ALL.

Key words: peri-transplantation minimal residual disease; risk stratification; risk scoring system; T-cell acute lymphoblastic leukemia
\end{abstract}

Disease recurrence remains one of the most common causes of death in patients with acute lymphoblastic leukemia (ALL) ${ }^{[1-6]}$, especially for those with T-cell ALL (T-ALL) ${ }^{[7]}$, who underwent allogeneic hematopoietic stem cell transplantation (allo-HSCT). Presently, a number of variables ${ }^{[2,7-15]}$, such as remission status pre-HSCT, immunophenotype of ALL, minimal/measurable residual disease (MRD)

Zhi-dong WANG, E-mail: wangzd73318@163.com; Yuewen WANG, E-mail: 527068345@qq.com

${ }^{\dagger}$ These two authors contributed equally to this work.

${ }^{\#}$ Corresponding author, E-mail: rmcyj@bjmu.edu.cn

${ }^{*}$ This work was supported (in part) by grants from the Beijing Municipal Science and Technology Commission (No. Z181100009618032), and the National Natural Science Foundation of China (Nos.: 81870141, 82070185, 81670186). and chronic graft-versus-host disease (GVHD), have been reported to be associated with relapse either in chemotherapy settings or in allo-HSCT modalities. Among these, MRD can be used not only for relapse prediction $^{[2,15,16]}$ but also for treatment selection ${ }^{[3,15,17]}$. Unfortunately, due to $10 \%-15 \%$ of total ALL cases were T-ALL ${ }^{[18]}$ most studies about the correlation of MRD with clinical outcomes focused on total ALL patients or those with B-cell ALL ${ }^{[2,3,11,12,15,16,19-21]}$. For patients with T-ALL, the effects of positive MRD at day 15 and 29 after therapy ${ }^{[7,22]}$, post-induction ${ }^{[6,23]}$ as well as pre-HSCT ${ }^{[2,10,24]}$ on relapse and survival have been previously demonstrated. However, there are limited data on the effects of peri-HSCT MRD on outcomes for ALL patients ${ }^{[12,20,25]}$ particularly for T-ALL patients. Therefore, we performed a retrospective study to investigate the effects of dynamic peri-HSCT MRD on 
transplantation outcomes in patients with T-ALL. In addition, we also tried to establish a risk score principally based on the dynamic peri-HSCT MRD combined with other parameters, such as remission status pre-HSCT and onset of chronic GVHD demonstrated by others ${ }^{[12,26,27]}$ and $\mathrm{us}^{[2,16,19]}$, which might provide better relapse risk determination for T-ALL patients.

\section{PATIENTS AND METHODS}

\subsection{Study Design}

This retrospective study included T-ALL subjects who were enrolled at the Peking University People's Hospital between January 2010 and December 2018. For patients with human leukocyte antigen (HLA)matched sibling donors (MSDs), MSDs were chosen. If cases without MSDs, HLA-matched unrelated donors (MUDs) were chosen. If cases without MSDs and MUDs, then haploidentical donors were chosen ${ }^{[28,29]}$. All of the included subjects signed an informed consent form. The study protocol was in accordance with the Declaration of Helsinki and was approved by the Institutional Review Board of Peking University. All of the cases were treated according to the transplant protocol as previously described ${ }^{[1,17,30]}$.

\subsection{Transplant Procedures}

Recombinant human granulocyte colonystimulating factor (G-CSF; $5 \mu \mathrm{g} / \mathrm{kg}$ per day for 5 days) were administered to healthy donors for bone marrow stem cells (BMSCs, collected on day 4 after G-CSF) and peripheral blood stem cells (PBSCs, collected on day 5 after G-CSF) mobilization ${ }^{[21,30]}$. Subjects received BMSCs and/or PBSCs as allografts.

All patients were treated with a myeloablative conditioning regimen ${ }^{[1,17,21,30]}$. For patients with haploidentical donors (HIDs), the conditioning regimen was given as follows: cytarabine $\left(4 \mathrm{~g} / \mathrm{m}^{2}\right.$ per day) intravenously on days -10 to -9 ; busulfan $(3.2 \mathrm{mg} / \mathrm{kg}$ per day) intravenously on days -8 to -6 ; cyclophosphamide $\left(1.8 \mathrm{~g} / \mathrm{m}^{2}\right.$ per day), intravenously on days -5 to -4 ; Me-CCNU $\left(250 \mathrm{mg} / \mathrm{m}^{2}\right.$ per day), orally once on day -3 ; and ATG (thymoglobulin, $2.5 \mathrm{mg} / \mathrm{kg}$ per day, Sang Stat, France) intravenously on days -5 to -2 . Patients with MSDs received hydroxycarbamide $(80 \mathrm{mg} / \mathrm{kg}$ ) orally on day -10 and a lower dose of cytarabine ( $2 \mathrm{~g} / \mathrm{m}^{2}$ per day) on day -9 , but otherwise, an identical regimen to the HID patients without ATG was employed.

\subsection{MFC Detection of MRD}

Bone marrow aspirate samples were obtained as part of the baseline assessment before SCT, as well as $1,2,3,4.5,6,9$, and 12 months posttransplantation and at 6-month intervals thereafter according to previous studies $^{[2,16,21,30]}$. Six- to eight-color MFC was performed for MRD evaluation according to previous studies ${ }^{[2,16,21]}$. A panel of antibody combinations recognizing cTdT,
mCD3, cCD3, CD5, CD7, CD34, CD45, and CD2 or CD99 was used for MRD determination. Any measurable level of MRD was considered positive, otherwise was defined as negative. The definition for quantitative dynamics of pre-MRD and postMRD included: (1) post-MRD increase after allograft compared with the pre-HSCT baseline; (2) post-MRD non-increase was defined as not meeting the criteria of (1) and (3); (3) unchanged negative MRD pre- and post-HSCT.

\subsection{Methods for MRD Intervention and Relapse Treatment}

Donor lymphocyte infusion (DLI) was performed as described previously by our group ${ }^{[1,17,31]}$. Other methods for positive MRD intervention and relapse treatment, such as interferon- $\gamma$ (IFN- $\gamma)$, were administered according to our previous studies ${ }^{[1,17,31]}$.

\subsection{Outcomes}

The primary study end point was the cumulative incidence of leukemia relapse. The secondary end points were the cumulative incidence of non-relapse mortality (NRM) and the probabilities of leukemia-free survival (LFS) and overall survival (OS).

The engraftment, infection, NRM, relapse, LFS, and OS were defined according to our previous studies $^{[1,17,31,32]}$. The definition and grades of acute GVHD were based on the pattern and severity of organ involvement ${ }^{[32]}$. The chronic GVHD was defined and graded according to the National Institute of Health criteria $^{[33]}$.

\subsection{Statistical Analysis}

Patient characteristics were compared between the negative MRD and positive MRD groups with the $\chi^{2}$ statistic for categorical variables and the MannWhitney test for continuous variables. Cumulative incidence curves were used in a competing risk setting, with relapse treated as a competing event to calculate NRM probabilities, and with death from any cause as a competing risk for GVHD, engraftment, and relapse. The probabilities of LFS and OS were estimated with the Kaplan-Meier method. The variables in table 1 were included in the univariate analysis. Only variables with $P<0.1$ were included in a Cox proportional hazards model with time-dependent variables. We calculated C-statistics (c), whereby a c value of 1.0 indicates perfect discrimination, and a c value of 0.5 is equivalent to chance ${ }^{[34]}$. Unless otherwise specified, $P$ values were based on two-sided hypothesis tests. Alpha was set at 0.05 . Most analyses were performed with SPSS software, version 16.0 (Mathsoft, USA).

\section{RESULTS}

\subsection{Patient Characteristics and Transplant Outcomes}

A total of 271 consecutive cases were included in this study. The characteristics of all of the cases, 


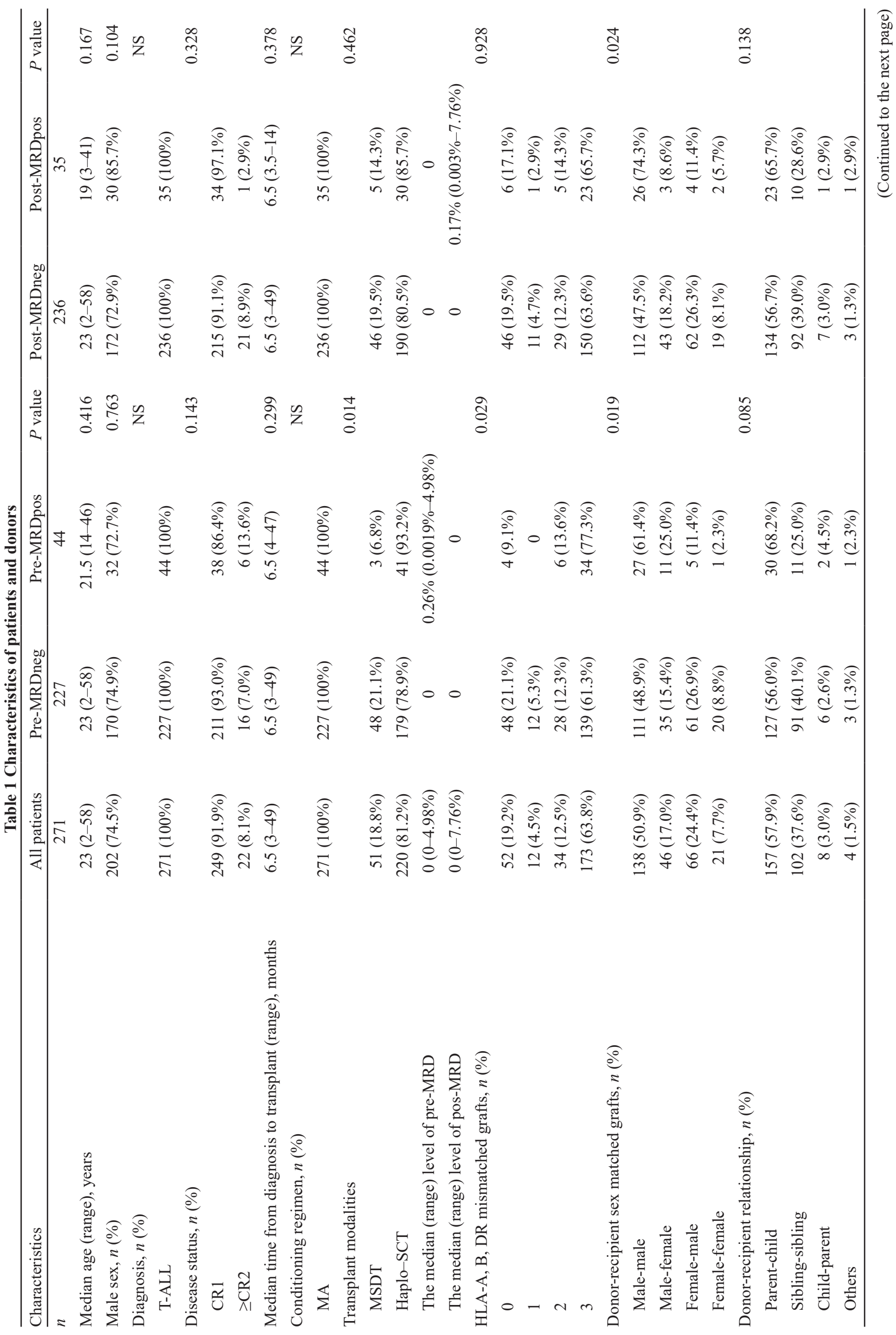


including positive and negative pre-HSCT or postHSCT MRD groups, are summarized in table 1. There was higher percentage of cases in the positive pre-HSCT MRD group receiving haploidentical HSCT than in the negative pre-HSCT group $(P=0.014)$. However, our previous studies demonstrated that, for ALL patients or acute myeloid leukemia patients, haploidentical HSCT

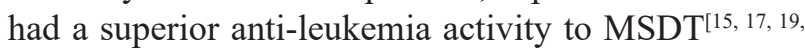
${ }^{35}$. Higher percentages of patients in the positive MRD groups received IFN- $\gamma$ or DLI for relapse intervention than those of the negative MRD groups either pre- or post-HSCT ( $P=0.030$ or $<0.001$, respectively) (table $1)$.

All patients had sustained, full donor chimerism. The cumulative 100-day incidence of platelet engraftment and acute GVHD grades II to IV was 93\% (95\% CI, 90\%-96\%) and 22\% (95\% CI, 17\%-27\%), respectively. After a median follow-up of 32 months (range, 0.93-127 months) and 52 months (range, 19127 months), for all cases and live cases, respectively, the 3-year cumulative incidence of chronic GVHD was $49 \%$ (95\% CI, 42\%-56\%). The 3-year NRM and cumulative incidence of relapse (CIR) were $13 \%$ (95\% CI, 9\%-17\%) and 26\% (95\% CI, 21\%-31\%), respectively. The 3-year probabilities of LFS and OS were $62 \%(95 \% \mathrm{CI}, 56 \%-68 \%)$ and $64 \%$ (95\% CI, $58 \%-70 \%$ ), respectively.

\subsection{Effects of Peri-HSCT MRDs on Outcomes}

We first investigated the effects of pre-HSCT MRDs or post-pre-HSCT MRDs on transplant outcomes in total cases. The patients in the positive pre-HSCT MRD group experienced higher CIR (45\% vs. $22 \%, P=0.003$ ), lower LFS (44\% vs. $65 \%$, $P=0.012)$ and $\mathrm{OS}(50 \%$ vs. $67 \%, P=0.051)$ than those in the negative pre-HSCT MRD group, but there was no significant difference in NRM between the positive and negative pre-HSCT MRD groups ( $11 \%$ vs. $13 \%$, $P=0.805)$. Patients with positive post-HSCT MRDs had higher CIR ( $72 \%$ vs. $19 \%, P<0.001)$, lower LFS $(22 \%$ vs. $67 \%, P<0.001)$ and OS $(32 \%$ vs. $69 \%$, $P<0.001)$ than those with negative post-HSCT MRDs, but no significant difference was found in NRM ( $6 \%$ vs. $14 \%, P=0.193$ ) (fig. 1 and table 2). Multivariate analysis showed that both positive pre-HSCT MRD and positive post-HSCT MRD were independently associated with leukemia relapse $(\mathrm{HR}=1.932,95 \% \mathrm{CI}$, 1.117-3.342, $P=0.019$ and $\mathrm{HR}=4.547,95 \% \mathrm{CI}, 2.717-$ 7.608, $P<0.001$, respectively) and LFS (HR $=1.932$, $95 \% \mathrm{CI}, 1.117-3.342, P=0.019$ and $\mathrm{HR}=2.920,95 \% \mathrm{CI}$, 1.848-4.613, $P<0.001$, respectively), but only positive post-HSCT MRD was correlated with OS (HR=2.448, 95\% CI, 1.525-3.931, $P<0.001)$. In addition, onset of chronic GVHD was associated with leukemia relapse (HR $=0.399,95 \%$ CI, 0.238-0.670, $P=0.001)$ and LFS (HR $=0.357,95 \%$ CI, $0.231-0.553, P<0.001)$.

We further evaluated the effects of peri-HSCT 

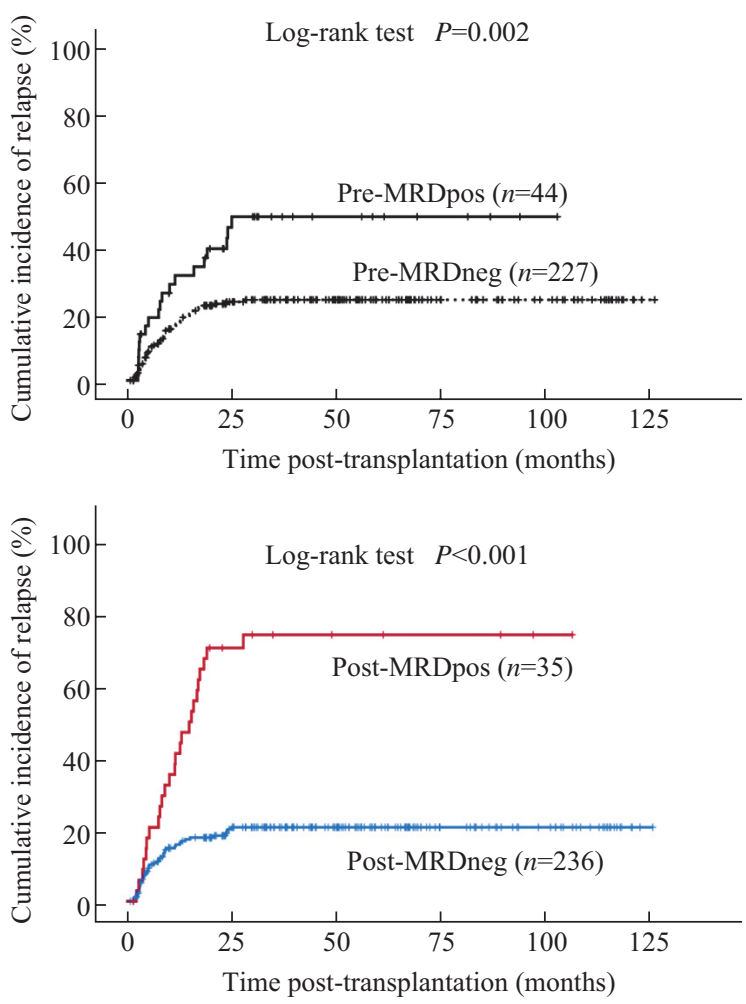

E

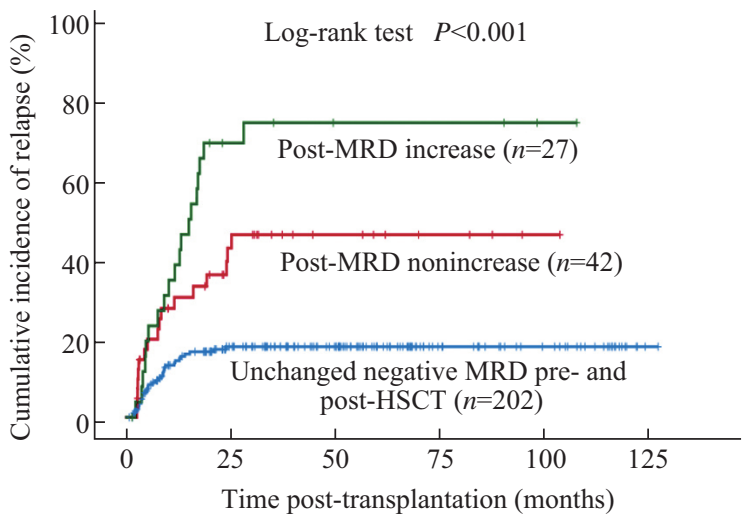

$\mathrm{G}$

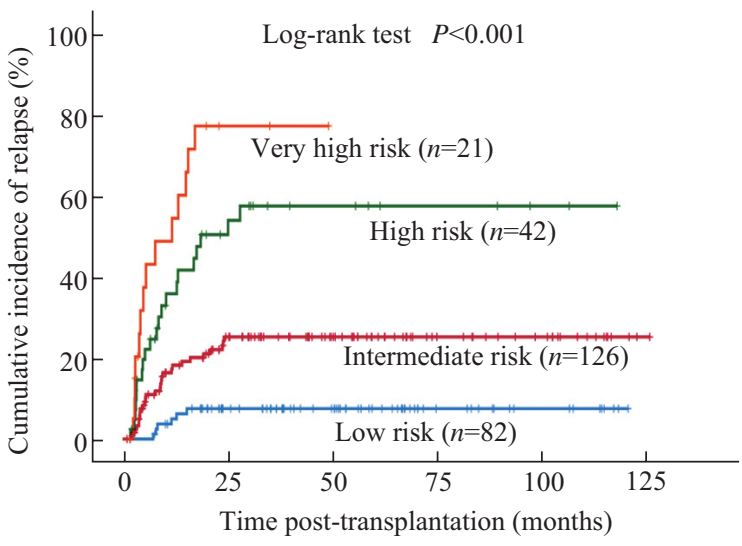

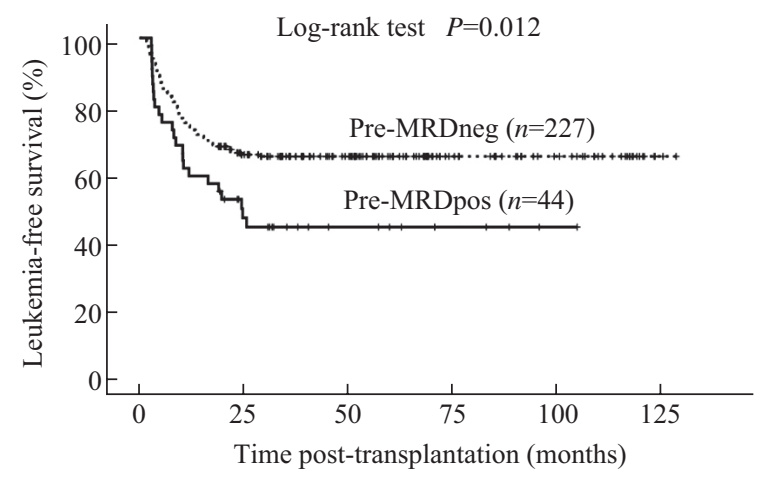

D

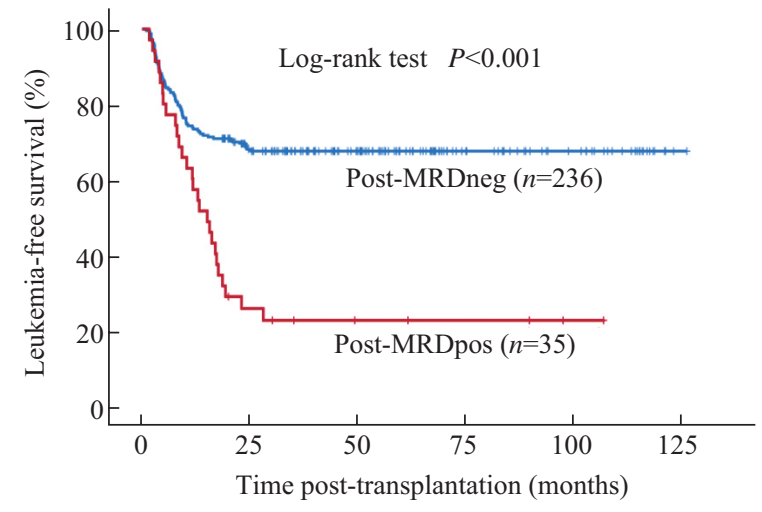

$\mathrm{F}$

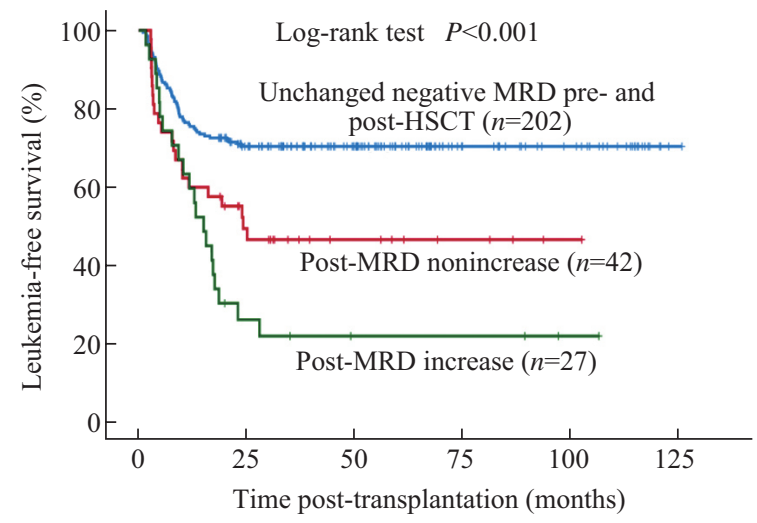

$\mathrm{H}$

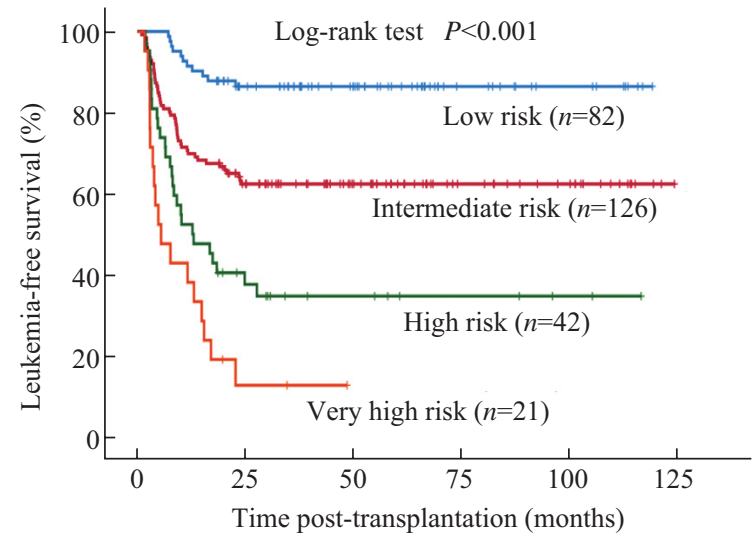

Fig. 1 Outcomes of T-ALL patients who underwent allogeneic stem cell transplantation according to different prognostic variables Cumulative incidence of 3-year relapses according to pre-HSCT MRD (A), post-HSCT MRD (C), dynamic peri-HSCT MRD (E), and risk scores $(\mathrm{G})$ principally based on dynamic peri-HSCT MRD. Probabilities of 3-year leukemia-free survival according to pre-HSCT MRD (B), post-HSCT MRD (D), dynamic peri-HSCT MRD (F), and risk scores (H) principally based on dynamic peri-HSCT MRD

T-ALL: T cell acute lymphoblastic leukemia; HSCT: hematopoietic stem cell transplantation; MRD: minimal/measurable residual disease 


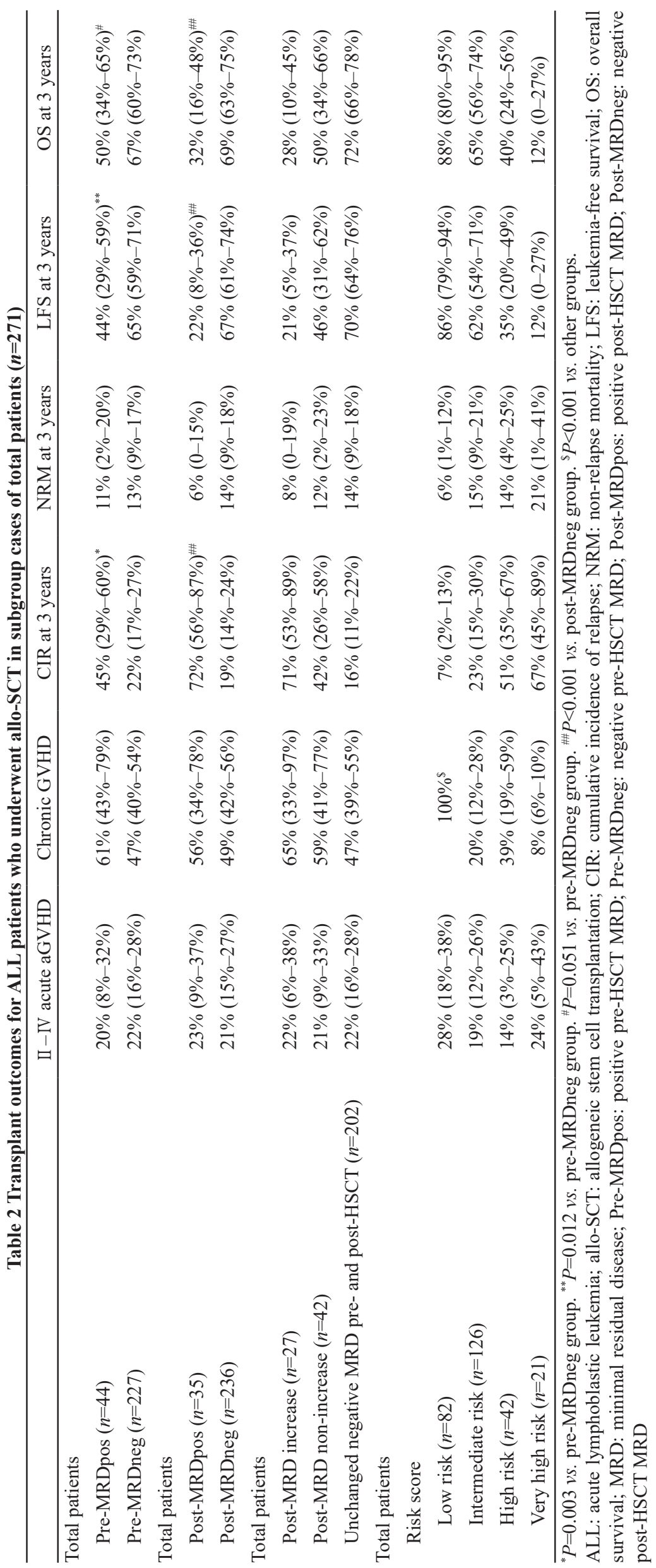


MRD on transplant outcomes. The total cases were classified as unchanged negative MRD pre- and postHSCT group (group A), post-MRD non-increase group (group B), and post-MRD increase group (group C), respectively. The CIR in group $\mathrm{B}$ and group $\mathrm{C}$ was significantly higher $(42 \%$ vs. $71 \%$ vs. $16 \%, P<0.001)$, LFS was significantly lower $(46 \%$ vs. $21 \%$ vs. $70 \%$, $P<0.001)$ and OS lower $(50 \%$ vs. $28 \%$ vs. $72 \%$, $P<0.001$ ), but NRM was comparable among three groups (14\% vs. $12 \%$ vs. $8 \%, P=0.752)$ (fig. 1 and table 2). Multivariate analysis showed that peri-HSCT MRD was associated with CIR $(\mathrm{HR}=2.392,95 \% \mathrm{CI}$, 1.816-3.151, $P<0.001)$, LFS (HR $=1.964,95 \% \mathrm{CI}$, 1.546-2.496, $P<0.001)$ and $\mathrm{OS}(\mathrm{HR}=1.731,95 \% \mathrm{CI}$, 1.348-2.222, $P<0.001$ ) (table 3). In addition, onset of chronic GVHD was associated with leukemia relapse (HR $=0.377,95 \%$ CI, $0.223-0.637, P<0.001)$ and LFS (HR $=0.344,95 \%$ CI, $0.222-0.535, P<0.001)$. The predictive value of peri-HSCT MRD on outcomes was also observed after analysis in the adult and pediatric subgroups (data not shown).

\subsection{A Risk Score for CIR Prediction}

We applied multivariate Cox regression analysis with stepwise forward selection based on the data of total patients. The final model included remission status before transplantation (CR1 scores: 0 ; $\geq \mathrm{CR} 2$ scores: 1 ), onset of chronic GVHD (with chronic GVHD scores: 0 ; without chronic GVHD scores: 1) and dynamics of pre- and post-HSCT MRD (pre-MRDneg and postMRDneg, MRD non-increase, and MRD increase, scores: 0,1 , and 2, respectively). According to the risk score categories, we classified each patient into one of four prognostic risk groups: low-risk ( $(\mathrm{score}=0$ ), intermediate-risk (score $=1)$, high-risk $($ score $=2)$ and very high-risk (score $=3$ ).

The 3-year CIR $(7 \%, 23 \%, 51 \%$, and $67 \%$, respectively, $P<0.001)$, NRM $(6 \%, 15 \%, 14 \%$, and $21 \%$, respectively, $P<0.001)$, LFS $(86 \%, 62 \%, 35 \%$, and $12 \%$, respectively, $P<0.001)$ and OS $(88 \%, 65 \%$, $40 \%$, and $12 \%$, respectively, $P<0.001$ ) in the low-risk, intermediate-risk, high-risk and very high-risk groups were listed in table 2 and fig. 1. Multivarate analysis

Table 3 Univariate and multivariate analysis of factors associated with outcomes of ALL patients who underwent allo-SCT considering dynamic peri-transplantation MRD $(n=271)$

\begin{tabular}{|c|c|c|c|c|c|c|}
\hline \multirow{2}{*}{ Covariate } & \multicolumn{3}{|c|}{ Univariate analysis } & \multicolumn{3}{|c|}{ Multivariate analysis } \\
\hline & HR & $95 \% \mathrm{CI}$ & $P$ value & HR & $95 \% \mathrm{CI}$ & $P$ value \\
\hline \multicolumn{7}{|l|}{ Relapse } \\
\hline Disease status $(\mathrm{CR} \geq 2$ vs. $\mathrm{CR} 1)$ & 2.929 & $1.450-5.918$ & 0.003 & 3.253 & $1.596-6.628$ & 0.001 \\
\hline Chronic GVHD (yes vs. no) & 0.434 & $0.260-0.726$ & 0.001 & 0.375 & $0.223-0.631$ & $<0.001$ \\
\hline Recipient age & 0.976 & $0.953-1.000$ & 0.047 & & & \\
\hline \multicolumn{7}{|l|}{ Dynamic peri-SCT MRD } \\
\hline MRD increase & 5.384 & $3.051-9.501$ & $<0.001$ & 6.076 & $3.420-10.792$ & $<0.001$ \\
\hline MRD non-increase & 2.923 & $1.627-5.249$ & $<0.001$ & 3.353 & $1.875-6.067$ & $<0.001$ \\
\hline Pre-MRDneg and post-MRDneg & & 1 & & & 1 & \\
\hline \multicolumn{7}{|l|}{ Non-relapse mortality } \\
\hline Disease status (CR $\geq 2$ vs. CR1) & 3.884 & $1.683-8.963$ & 0.001 & 4.621 & $1.960-10.892$ & $<0.001$ \\
\hline Platelet engraftment & 2.875 & $1.244-6.562$ & 0.013 & 2.905 & $1.263-6.681$ & 0.012 \\
\hline Neutrophil engraftment & 1.809 & $0.895-3.656$ & 0.099 & & & \\
\hline Recipient age & 1.028 & $0.997-1.059$ & 0.074 & 2.068 & $1.026-4.170$ & 0.042 \\
\hline \multicolumn{7}{|l|}{ Leukemia-free survival } \\
\hline Disease status ( $\mathrm{CR} \geq 2$ vs. $\mathrm{CR} 1)$ & 3.494 & $2.044-5.972$ & $<0.001$ & 3.598 & $2.093-6.185$ & $<0.001$ \\
\hline Gender (female $v s$. male) & 0.565 & $0.340-0.940$ & 0.028 & & & \\
\hline Chronic GVHD (yes vs. no) & 0.375 & $0.243-0.579$ & $<0.001$ & 0.344 & $0.222-0.535$ & $<0.001$ \\
\hline \multicolumn{7}{|l|}{ Dynamic peri-SCT MRD } \\
\hline MRD increase & 3.202 & $1.944-5.275$ & $<0.001$ & 3.685 & $2.225-6.104$ & $<0.001$ \\
\hline MRD non-increase & 2.057 & $1.261-3.535$ & 0.004 & 2.358 & $1.439-3.863$ & 0.001 \\
\hline Pre-MRDneg and post-MRDneg & & 1 & & & 1 & \\
\hline \multicolumn{7}{|l|}{ Overall survival } \\
\hline Disease status $(\mathrm{CR} \geq 2$ vs. $\mathrm{CR} 1)$ & 3.585 & $2.088-6.157$ & $<0.001$ & 3.676 & $2.137-6.322$ & $<0.001$ \\
\hline Gender (female vs. male) & 0.589 & $0.348-0.995$ & 0.048 & & & \\
\hline \multicolumn{7}{|l|}{ Dynamic peri-SCT MRD } \\
\hline MRD increase & 2.803 & $1.663-4.723$ & $<0.001$ & 2.922 & $1.732-4.929$ & $<0.001$ \\
\hline MRD non-increase & 1.943 & $1.165-3.238$ & 0.011 & 1.891 & $1.133-3.145$ & 0.015 \\
\hline Pre-MRDneg and post-MRDneg & & 1 & & & 1 & \\
\hline
\end{tabular}

${ }^{*}$ All variables were first included in the univariate analysis; only variables with $P<0.1$ were included in the Cox proportional hazards model with time-dependent variables.

ALL: acute lymphoblastic leukemia; Allo-SCT: allogeneic stem cell transplantation; MRD: minimal/measurable residual disease; HR: hazard ratio; CI: confidence interval; CR: complete remission; GVHD: graft-versus-host disease; peri-SCT: peri-stem cell transplantation; pre-MRDneg: negative pre-transplantation MRD; post-MRDneg: negative post-transplantation MRD 
showed that the risk score was associated with CIR $(\mathrm{HR}=2.574,95 \% \mathrm{CI}, 2.013-3.291, P<0.001), \mathrm{NRM}$ $(\mathrm{HR}=1.734,95 \% \mathrm{CI}, 1.198-2.508, P=0.004)$, LFS $(\mathrm{HR}=2.229,95 \% \mathrm{CI}, 1.822-2.728, P<0.001)$ and $\mathrm{OS}$ $(\mathrm{HR}=2.164,95 \% \mathrm{CI}, 1.755-2.667, P<0.001)$ (table 4). The risk score system could better distinguish CIR $(c=0.730)$ than that for pre-HSCT MRD $(c=0.562)$, post-HSCT MRD $(\mathrm{c}=0.616)$ and pre- and post-MRD dynamics $(c=0.648)$. The value of the risk score for CIR prediction was also observed after analysis in the adult and pediatric subgroups (data not shown).

\section{DISCUSSION}

In consistent with previous studies either in total ALL patients $^{[16,20]}$ or B-ALL subgroup patients ${ }^{[36]}$, we, in the present study, found that dynamic peri-HSCT MRD in patients with T-ALL could be better for discrimination of relapse risk than that of pre-HSCT MRD or posHSCT MRD. In addition, we showed that a risk score principally based on dynamic peri-transplantation MRD could further achieve better relapse stratification than dynamic peri-HSCT MRD alone (c-index $=0.730$ vs. 0.648). Overall, our results add new evidence for the application of MRD, suggesting the usefulness of dynamic peri-HSCT MRD for stratification of T-ALL patients with high risk recurrence.

In a recent study including 477 B-ALL patients who underwent allo-HSCT, Cao et a ${ }^{[19]}$ demonstrated that post-HSCT MRD, but not pre-HSCT MRD was associated with higher CIR and shorter survival after multivariate analysis. In contrast to the result by Cao et $a l^{[19]}$, we found that both pre- and post-MRD could be used for discriminating patients into different relapse risk groups for T-ALL patients, although post-HSCT MRD was better than pre-HSCT MRD in predicting leukemia relapse (table 2 and fig. 1). The abovementioned differences might be related to the higher CIR and shorter survival in patients with T-ALL who underwent allografts than in B-ALL patients who

Table 4 Univariate and multivariate analysis of factors associated with outcomes of ALL patients who underwent allo-SCT considering a risk score based on dynamic peri-transplantation MRD $(n=271)$

\begin{tabular}{|c|c|c|c|c|c|c|}
\hline \multirow{2}{*}{ Covariate } & \multicolumn{3}{|c|}{ Univariate analysis } & \multicolumn{3}{|c|}{ Multivariate analysis } \\
\hline & HR & $95 \% \mathrm{CI}$ & $P$ value & HR & $95 \% \mathrm{CI}$ & $P$ value \\
\hline \multicolumn{7}{|l|}{ Relapse } \\
\hline Recipient age & 0.976 & $0.953-1.000$ & 0.047 & & & \\
\hline \multicolumn{7}{|l|}{ Risk score } \\
\hline Low risk & & 1 & & & 1 & \\
\hline Intermediate risk & 3.670 & $1.519-8.865$ & 0.004 & 3.670 & $1.519-8.865$ & 0.004 \\
\hline High risk & 10.309 & $4.154-25.583$ & $<0.001$ & 10.309 & $4.154-25.583$ & $<0.001$ \\
\hline Very high risk & 19.412 & $7.417-50.803$ & $<0.001$ & 19.412 & $7.417-50.803$ & $<0.001$ \\
\hline \multicolumn{7}{|l|}{ Non-relapse mortality } \\
\hline Platelet engraftment & 2.875 & $1.244-6.562$ & 0.013 & 3.331 & $1.438-7.717$ & 0.005 \\
\hline Neutrophil engraftment & 1.809 & $0.895-3.656$ & 0.099 & & & \\
\hline Recipient age & 1.028 & $0.997-1.059$ & 0.074 & 1.036 & $1.004-1.069$ & 0.027 \\
\hline \multicolumn{7}{|l|}{ Risk score } \\
\hline Low risk & & 1 & & & 1 & \\
\hline Intermediate risk & 2.792 & $1.042-7.479$ & 0.041 & 3.285 & $1.217-8.870$ & 0.019 \\
\hline High risk & 2.802 & $0.854-9.193$ & 0.089 & 3.282 & $0.990-10.881$ & 0.052 \\
\hline Very high risk & 4.381 & $1.172-16.384$ & 0.028 & 7.463 & $1.950-28.349$ & 0.003 \\
\hline \multicolumn{7}{|l|}{ Leukemia-free survival } \\
\hline Gender (female vs. male) & 0.565 & $0.340-0.940$ & 0.028 & & & \\
\hline \multicolumn{7}{|l|}{ Risk score } \\
\hline Low risk & & 1 & & & 1 & \\
\hline Intermediate risk & 3.333 & $1.728-6.246$ & $<0.001$ & 3.333 & $1.728-6.246$ & $<0.001$ \\
\hline High risk & 7.058 & $3.496-14.294$ & $<0.001$ & 7.058 & $3.496-14.294$ & $<0.001$ \\
\hline Very high risk & 12.928 & $6.077-27.503$ & $<0.001$ & 12.928 & $6.077-27.503$ & $<0.001$ \\
\hline \multicolumn{7}{|l|}{ Overall survival } \\
\hline Gender (female vs. male) & 0.589 & $0.348-0.995$ & 0.048 & & & \\
\hline \multicolumn{7}{|l|}{ Risk score } \\
\hline Low risk & & 1 & & & 1 & \\
\hline Intermediate risk & 3.293 & $1.655-6.554$ & 0.001 & 3.293 & $1.655-6.554$ & 0.001 \\
\hline High risk & 6.290 & $3.004-13.167$ & $<0.001$ & 6.290 & $3.004-13.167$ & $<0.001$ \\
\hline Very high risk & 12.364 & $5.677-26.927$ & $<0.001$ & 12.364 & $5.677-26.927$ & $<0.001$ \\
\hline
\end{tabular}

*All variables were first included in the univariate analysis; only variables with $P<0.1$ were included in the Cox proportional hazards model with time-dependent variables.

ALL: acute lymphoblastic leukemia; Allo-SCT: allogeneic stem cell transplantation; MRD: minimal/measurable residual disease; HR: hazard ratio; $\mathrm{CI}$ : confidence interval 
received allo-HSCT ${ }^{[2]}$. However, the results of the present study are consistent with those of previous study including both T-ALL and B-ALL cases receiving allograft, reported by Bader et a ${ }^{[12]}$. They reported a higher c-index for the post-HSCT MRD than that of pre-HSCT MRD (c-index $=0.649$ vs. 0.612).

Considering MRD was detected at different time points, several studies focused on the dynamic MRD change in predicting CIR for patients with acute leukemia $^{[12,16,20,25,37,38]}$. For 279 patients with AML receiving allografts, pre-HSCT MRD and post-HSCT MRD were determined by MFC. Zhou et al ${ }^{[38]}$ found that patients with increased MRD levels around the time of transplantation experienced higher CIR and shorter survival than those with decreased MRD levels or those with negative pre-HSCT MRD and negative post-HSCT MRD. We have also confirmed the results reported by Zhou et al[38] either in total AML patients or in pediatric and young adult ALL cases. Here, we confirmed the superiority of dynamic peri-HSCT MRD compared to pre-HSCT MRD and post-HSCT MRD in T-ALL patients receiving allo-HSCT (table 2 and fig. 1). The results of our previous study ${ }^{[16]}$ and other studies $^{[20,38]}$ suggest that dynamic peri-HSCT MRD should be routinely used for CIR discrimination in allograft settings for patients with acute leukemia.

In a large cohort of study including 616 pediatric and young adult ALL patients receiving allografts, Bader et $a l^{[12]}$ showed that these patients could be classified into three different relapse risk groups according to a prognostic risk score established based on remission status before transplantation, conditioning regimen and pre-HSCT MRD. In the present study, except for dynamic MRD peri-transplantation, remission status pre-HSCT and onset of chronic GVHD were also independently correlated with CIR and survival. We found, in subgroup patients with T-ALL who underwent allograft, a risk score principally based on dynamic peri-HSCT MRD as well as remission status and chronic GVHD could further classify patients into four subgroups with different CIR and survival (table 2 and fig. 1). Therefore, the results in the present study and previous studies shown by others ${ }^{[12,39]}$ and us ${ }^{[36]}$ suggest that risk scores based on MRD and other variables could be better in predicting transplant outcomes for ALL patients, especially those of T-ALL with a CIR more than 50\% (table 2 and fig. 1).

There are limitations of our study. First, this is a retrospective, single center study. Second, the present study only enrolled T-ALL patients who underwent haploidentical HSCT and MSDT. Third, we did not perform subgroup analysis of cases who received MSDT due to the small number of patients in subgroup. Therefore, a prospective, multicenter study with training and validation sets is needed to further confirm whether our findings are suitable for T-ALL cases who either received haploidentical HSCT (including haploidentical HSCT based on immune tolerance induced by post-transplantation cyclophosphamide), MSDT, MUD transplantation ${ }^{[5]}$ or umbilical cord blood transplantation ${ }^{[40]}$.

In summary, our results suggest a superiority of dynamic peri-HSCT MRD to single time point, including pre-HSCT MRD and post-HSCT MRD, in relapse risk stratification for patients with T-ALL. We further suggest that T-ALL patients who would experience the worst outcome could be discriminated by a risk score principally based on dynamic periHSCT MRD, for these cases, novel strategies are needed to improve the transplant outcomes.

\section{Acknowledgement}

We would like to thank Miss Xu Wu for her assistance in minimal residual disease detection.

\section{Open Access}

This article is licensed under a Creative Commons Attribution 4.0 International License https://creativecommons.org/licenses/by/4.0/), which permits use, sharing, adaptation, distribution and reproduction in any medium or format, as long as you give appropriate credit to the original author(s) and the source, provide a link to the Creative Commons licence, and indicate if changes were made. The images or other third party material in this article are included in the article's Creative Commons licence, unless indicated otherwise in a credit line to the material. If material is not included in the article's Creative Commons licence and your intended use is not permitted by statutory regulation or exceeds the permitted use, you will need to obtain permission directly from the copyright holder. To view a copy of this licence, visit http://creativecommons. org/licenses/by/4.0/.

\section{Conflict of Interest Statement}

The authors declare no conflict of interest.

\section{REFERENCES}

1 Wang Y, Liu QF, Xu LP, et al. Haploidentical versus Matched-Sibling Transplant in Adults with PhiladelphiaNegative High-Risk Acute Lymphoblastic Leukemia: A Biologically Phase III Randomized Study. Clin Cancer Res, 2016,22(14):3467-3476

2 Zhao XS, Liu YR, Xu LP, et al. Minimal residual disease status determined by multiparametric flow cytometry pretransplantation predicts the outcome of patients with ALL receiving unmanipulated haploidentical allografts. Am J Hematol, 2019,94(4):512-521

3 Pui CH, Pei D, Coustan-Smith E, et al. Clinical utility of sequential minimal residual disease measurements in the context of risk-based therapy in childhood acute lymphoblastic leukaemia: a prospective study. Lancet Oncol, 2015,16(4):465-474

4 Sun YQ, Li SQ, Zhao XS,et al. Measurable residual disease of acute lymphoblastic leukemia in allograft settings: how to evaluate and intervene. Expert Rev Anticancer Ther, 2020,20(6):453-564 
5 Chang YJ, Zhao XY, Huang XJ. Granulocyte Colony-Stimulating Factor-Primed Unmanipulated Haploidentical Blood and Marrow Transplantation. Front Immunol, 2019, 10:2516

6 Hay KA, Gauthier J, Hirayama AV, et al. Factors associated with durable EFS in adult B-cell ALL patients achieving MRD-negative CR after CD19 CAR T-cell therapy. Blood, 2019,133(15):1652-1663

7 Modvig S, Madsen HO, Siitonen SM, et al. Minimal residual disease quantification by flow cytometry provides reliable risk stratification in T-cell acute lymphoblastic leukemia. Leukemia, 2019,33(6):13241336

8 Malard F, Mohty M. Acute lymphoblastic leukaemia. Lancet, 2020,395(10230):1146-1162

9 Burns MA, Place AE, Stevenson KE, et al. Identification of prognostic factors in childhood T-cell acute lymphoblastic leukemia: Results from DFCI ALL Consortium Protocols 05-001 and 11-001. Pediatr Blood Cancer, 2021,68(1):e28719

$10 \mathrm{Xu} \mathrm{M}$, Liu $\mathrm{H}$, Liu $\mathrm{Y}$, et al. Gene mutations and pretransplant minimal residual disease predict risk of relapse in adult patients after allogeneic hematopoietic stem-cell transplantation for T cell acute lymphoblastic leukemia. Leuk Lymphoma, 2019,60(11):2744-2753

11 Bader P, Kreyenberg H, von Stackelberg A, et al. Monitoring of minimal residual disease after allogeneic stem-cell transplantation in relapsed childhood acute lymphoblastic leukemia allows for the identification of impending relapse: results of the ALL-BFM-SCT 2003 trial. J Clin Oncol, 2015,33(11):1275-1284

12 Bader P, Salzmann-Manrique E, Balduzzi A, et al. More precisely defining risk peri-HCT in pediatric ALL: pre- vs post-MRD measures, serial positivity, and risk modeling. Blood Adv, 2019,3(21):3393-3405

13 Eckert C, Hagedorn N, Sramkova L, et al. Monitoring minimal residual disease in children with high-risk relapses of acute lymphoblastic leukemia: prognostic relevance of early and late assessment. Leukemia, 2015, 29(8):1648-1655

14 Zhao X, Hong Y, Qin Y, et al. The clinical significance of monitoring the expression of the SIL-TAL1 fusion gene in T-cell acute lymphoblastic leukemia after allogeneic hematopoietic stem cell transplantation. Int J Lab Hematol, 2017,39(6):613-619

15 Li SQ, Fan QZ, Xu LP, et al. Different Effects of Pre-transplantation Measurable Residual Disease on Outcomes According to Transplant Modality in Patients With Philadelphia Chromosome Positive ALL. Front Oncol, 2020,10:320

16 Wang XY, Fan QZ, Xu LP, et al. The Quantification of Minimal Residual Disease Pre- and Post-Unmanipulated Haploidentical Allograft by Multiparameter Flow Cytometry in Pediatric Acute Lymphoblastic Leukemia. Cytometry B Clin Cytom, 2020,98(1):75-87

17 Chang YJ, Wang Y, Xu LP, et al. Haploidentical donor is preferred over matched sibling donor for pretransplantation MRD positive ALL: a phase 3 genetically randomized study. J Hematol Oncol, 2020,13(1):27

18 Teachey DT, Pui CH. Comparative features and outcomes between paediatric T-cell and B-cell acute lymphoblastic leukaemia. Lancet Oncol, 2019,20(3):e142-e54
19 Cao LQ, Zhou Y, Liu YR, et al. A risk score system for stratifying the risk of relapse in $B$ cell acute lymphocytic leukemia patients after allogenic stem cell transplantation. Chin Med J (Engl), 2021,134(10):11991208

20 Lovisa F, Zecca M, Rossi B, et al. Pre- and posttransplant minimal residual disease predicts relapse occurrence in children with acute lymphoblastic leukaemia. Br J Haematol, 2018,180(5):680-693

21 Zhao XS, Liu YR, Zhu HH, et al. Monitoring MRD with flow cytometry: an effective method to predict relapse for ALL patients after allogeneic hematopoietic stem cell transplantation. Ann Hematol, 2012,91(2):183-192

22 Quist-Paulsen P, Toft N, Heyman M, et al. T-cell acute lymphoblastic leukemia in patients $1-45$ years treated with the pediatric NOPHO ALL2008 protocol. Leukemia, 2020,34(2):347-357

23 Tembhare PR, Narula G, Khanka T, et al. Post-induction Measurable Residual Disease Using Multicolor Flow Cytometry Is Strongly Predictive of Inferior Clinical Outcome in the Real-Life Management of Childhood T-Cell Acute Lymphoblastic Leukemia: A Study of 256 Patients. Front Oncol, 2020,10:577

24 Brammer JE, Saliba RM, Jorgensen JL, et al. Multicenter analysis of the effect of T-cell acute lymphoblastic leukemia subtype and minimal residual disease on allogeneic stem cell transplantation outcomes. Bone Marrow Transplant, 2017,52(1):20-27

25 Sutton R, Shaw PJ, Venn NC, et al. Persistent MRD before and after allogeneic BMT predicts relapse in children with acute lymphoblastic leukaemia. $\mathrm{Br} \mathrm{J}$ Haematol, 2015,168(3):395-404

26 Yeshurun M, Weisdorf D, Rowe JM, et al. The impact of the graft-versus-leukemia effect on survival in acute lymphoblastic leukemia. Blood Adv, 2019,3(4):670-680

27 Terwey TH, Le Duc TM, Hemmati PG, et al. NIHdefined graft-versus-host disease and evidence for a potent graft-versus-leukemia effect in patients with acute lymphoblastic leukemia. Ann Oncol, 2013,24(5):13631370

28 van Rood JJ, Oudshoorn M. When selecting an HLA mismatched stem cell donor consider donor immune status. Curr Opin Immunol, 2009,21(5):538-543

29 Ballen KK, Koreth J, Chen YB, et al. Selection of optimal alternative graft source: mismatched unrelated donor, umbilical cord blood, or haploidentical transplant. Blood, 2012,119(9):1972-1980

30 Huang XJ, Liu DH, Liu KY, et al. Treatment of acute leukemia with unmanipulated HLA-mismatched/ haploidentical blood and bone marrow transplantation. Biol Blood Marrow Transplant, 2009,15(2):257-265

31 Chang YJ, Xu LP, Wang Y, et al. Controlled, Randomized, Open-Label Trial of Risk-Stratified Corticosteroid Prevention of Acute Graft-Versus-Host Disease After Haploidentical Transplantation. J Clin Oncol, 2016,34(16):1855-1863

32 Chang YJ, Wu DP, Lai YR, et al. Antithymocyte Globulin for Matched Sibling Donor Transplantation in Patients With Hematologic Malignancies: A Multicenter, OpenLabel, Randomized Controlled Study. J Clin Oncol, 2020,38(29):3367-3376

33 Shulman HM, Cardona DM, Greenson JK, et al. NIH 
Consensus development project on criteria for clinical trials in chronic graft-versus-host disease: II. The 2014 Pathology Working Group Report. Biol Blood Marrow Transplant, 2015,21(4):589-603

34 International CLLIPIwg. An international prognostic index for patients with chronic lymphocytic leukaemia (CLL-IPI): a meta-analysis of individual patient data. Lancet Oncol, 2016,17(6):779-790

35 Guo H, Chang YJ, Hong Y, et al. Dynamic immune profiling identifies the stronger graft-versus-leukemia (GVL) effects with haploidentical allografts compared to HLA-matched stem cell transplantation. Cell Mol Immunol, 2021,18(5):1172-1185

36 Walter RB, Appelbaum FR. Next-generation sequencing for measuring minimal residual disease in AML. Nat Rev Clin Oncol, 2018,15(8):473-474

37 Liu J, Ma R, Liu YR, et al. The significance of peri- transplantation minimal residual disease assessed by multiparameter flow cytometry on outcomes for adult AML patients receiving haploidentical allografts. Bone Marrow Transplant, 2019,54(4):567-577

38 Zhou Y, Othus M, Araki D, et al. Pre- and post-transplant quantification of measurable ('minimal') residual disease via multiparameter flow cytometry in adult acute myeloid leukemia. Leukemia, 2016,30(7):14561464

39 Enshaei A, O'Connor D, Bartram J, et al. A validated novel continuous prognostic index to deliver stratified medicine in pediatric acute lymphoblastic leukemia. Blood, 2020,135(17):1438-1446

40 Gabelli M, Veys P, Chiesa R. Current status of umbilical cord blood transplantation in children. Br J Haematol, 2020,190(5):650-683

(Received Mar. 31, 2021; accepted Jun. 18, 2021) 